\title{
FEATURES OF THE FASTEST PRESSURE GROWTH POINT DURING COMPRESSION STROKE
}

\author{
Oleksiy YERYGANOV, Roman VARBANETS \\ Odessa National Maritime University, Mechnikov street, 34, Odessa, Ukraine, 65029 \\ office@,onmu.odessa.ua, a.yeryganov@gmail.com, office@,onmu.odessa.ua, roman.varbanets@gmail.com
}

\begin{abstract}
The article contains description of method, which enables to synchronize indicator diagram of diesel engine using the parameters of fastest pressure growth point during compression stroke. It means calculation of this point position relatively to top dead center (TDC). At first, experimentally obtained data has to be smoothed by the Butterworth low pass filter. Afterwards, assumed that the process is stationary, compression stroke is expressed by equation of polytropic compression. Then the first and the second pressure derivatives are determined by crank angle. The condition, when the second pressure derivative by crank angle equals zero, enables us to find the position of the fastest pressure growth point relatively to TDC. The angle, which was found in such a way, is used later as first approximation for finding the TDC position with adequate accuracy. It's also shown how to calculate the volume of compression chamber (compression ratio) using parameters of this point. Results of the calculation are compared with experimentally obtained data.
\end{abstract}

Keywords: top dead centre, point of fastest pressure growth, polytropic compression, pressure derivative by crank angle

\section{INTRODUCTION}

The indicator diagram of engine is one of the most important methods for assessment of its technical condition. It's very important that Top Dead Center position (TDC) has to be calculated with maximum accuracy. In the beginning of the analysis, TDC position is calculated preliminary and then - more precisely. Pressure data, received from indicator diagram, are taken for preliminary calculating of TDC position. For more precise calculating of TDC position there are lots of algorithms including those based on the entropy calculation [15] or on quantities of heat transferred from/to cylinder surface [13].

Error in TDC calculation can result in mistake for other values. For instance in case of $1 \%$ error of TDC calculating, the error for mean indicated pressure is $9 \%$ [7]. Analysis of values depending on error of TDC position was given in $[1,2,11]$.

\section{CALCULATING OF TDC POSITION}

\subsection{Choice of considering point}

Most of algorithmic TDC determination methods use the well-known fact: in case of absence of combustion and zero thermodynamic displacement $\left(\delta \varphi_{T}=0\right)$, the pressure in cylinder in TDC point runs up to its maximum, and its first derivative of the crank angle (CA) equals zero:

$$
\left.P\right|_{\varphi=\varphi_{\mathrm{TDC}}} \rightarrow \max \Rightarrow d P /\left.d \varphi\right|_{\varphi=\varphi_{\mathrm{TDC}}}=0 .
$$

On the other hand in most cases compression stroke of real indicator diagram does not contain the point $d P / d \varphi=0$ as combustion begins before TDC very often. That is why it is more logic in preliminary TDC calculations to use data of actual point really presented on the indicator diagram, because in this case it won't be necessary to find additional conditions and restrictions.

It is mentioned in [9], that maximum position of the first pressure derivative by $\mathrm{CA}$ in compression stroke is quite definite value, which is almost steady under running conditions. Under these conditions the polytropic coefficient varies within the limits 1.3-1.4.

Analysis shows that fuel combustion in most diesels begins at compression stroke after maximum of the first pressure derivative by CA. It means that this actual point presented in fact in the indicator diagram (and its parameters) can be taken for calculating preliminary location of TDC (i.e. in order to synchronize indicator diagram). By the meantime, it's necessary to keep in mind, that data for $d P / d \varphi$ are noisy and therefore has to be thoroughly filtrated.

\subsection{Data filtration}

To record and analyze indicator diagrams let us use IMES GmbH (Kaufbeuren, Germany) pressure sensors [3]. Despite high quality of German pressure sensors, their low relative error $(\sigma<0.5 \%)$ and low noise level, further numeric differentiation of the received indicator diagrams leads to practical impossibility of numeric analysis of curves $d P / d \varphi$. In this case determination of maximum $d P / d \varphi$ coordinates before the combustion, also will be carried out with rather big error (fig.1). It becomes obvious that it is necessary to apply the correct procedure for filtering the original data.

We prefer to use the Butterworth digital lowpass filter [12] because: its frequency characteristics are close to those of the ideal integrator, and are monotonic in the passband; besides it is most simple in implementation and description. 


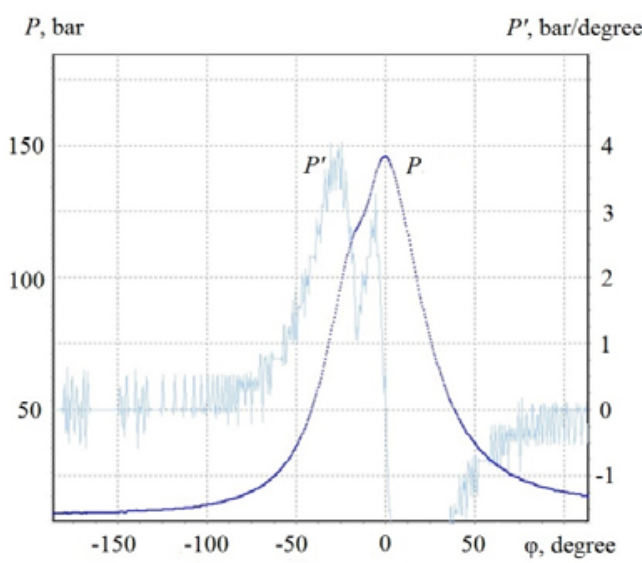

Fig. 1. Cylinder pressure $P(\varphi)$ and its derivative $P^{\prime}(\varphi)$ before filtration

Frequency characteristics of $j$-th order Butterworth digital low-pass filter are:

$$
G(f)=\frac{G_{0}}{\sqrt{1+\left(f / f_{C}\right)^{2 j}}},
$$

where $G_{0}$ - zero-frequency gain coefficient; $f_{C}$ - the cutoff frequency (at which the amplitude is $3 \mathrm{~dB}$ [12]); $j$ - the filter number (order).

The filter parameters can be selected/chosen using the recommendations [10].

Fig. 1 shows the $P(\varphi)$ and $P^{\prime}(\varphi)$ diagrams before the Butterworth low-pass filter was applied. Due to analog and digital noise' influence while recording $P(\varphi)$, determination of maximum $P^{\prime}(\varphi)$ coordinate will be made with a big error. The second $P^{\prime \prime}(\varphi)$ derivative analysis is just not possible in this case.

Application of the Butterworth low-pass filter makes it possible to analyze numerically the first $P^{\prime}(\varphi)$ and the second $P^{\prime \prime}(\varphi)$ derivatives (fig. 2), as well as to determine the coordinate of the maximum compression stroke speed in the working cylinder (point $P_{\mathrm{m}}^{\prime}$ ).

While recording indicator diagrams with the help of IMES GmbH pressure sensors (with relative error less than $0.5 \%$ and sampling step $\Delta \leq 0.5^{\circ}$ $\mathrm{CA}$ ), the filter order $j=5.5 \div 7$ provided enough quality of high-frequency noise suppression on $P^{\prime}(\varphi)$ and $P^{\prime \prime}(\varphi)$ curves. Besides it made it possible to perform their further analysis for local extremum, which were later used to provide indicator diagrams synchronization, i.e. to transfer them from the functions of time into the CA functions [16]:

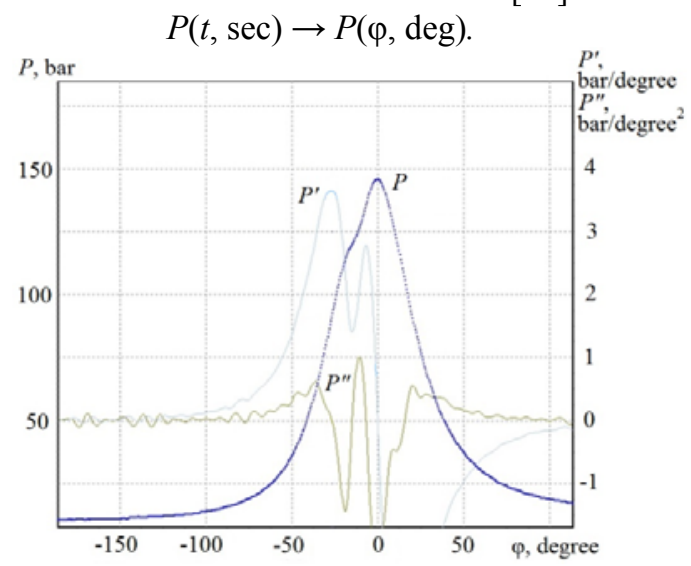

Fig. 2. Cylinder pressure $P(\varphi)$ and its derivatives $P^{\prime}(\varphi)$ and $P^{\prime \prime}(\varphi)$ after applying the Butterworth low pass
2.3. Analysis of the fastest pressure growth point

Let's analyze indicator diagram in the point of fastest pressure growth. In this point the value of the first pressure derivative runs up to its maximum $(d P / d \varphi \rightarrow \max )$. It means also that the second pressure derivative equals zero $\left(d^{2} P / d \varphi^{2}=0\right)$. This angle, where $d P / d \varphi \rightarrow \max$, we indicate as $\varphi_{\mathrm{m}}$. Presume that crank shaft rotating speed is known and steady, fuel injection rate is constant and compression of working medium in cylinder is polytropic $\left(P V^{n}=\right.$ const $)$. Let's find the second pressure derivative and equate it with zero.

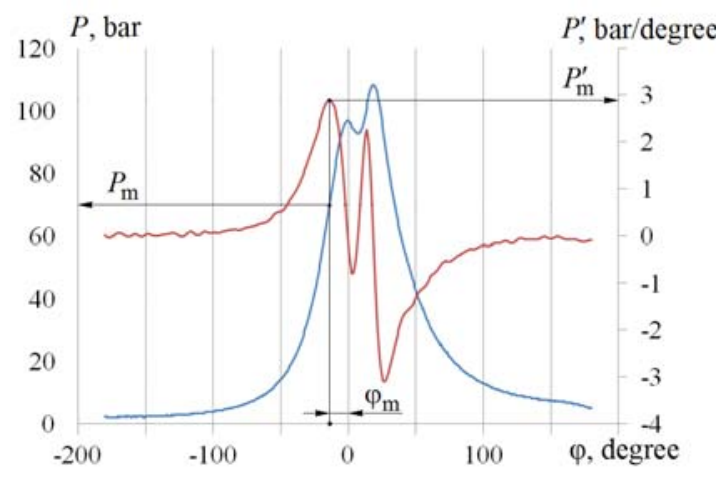

Fig. 3. Cylinder pressure and its derivative of the crank angle

Let us denote:

$$
\frac{d P}{d \varphi}=P^{\prime} ; \frac{d^{2} P}{d \varphi^{2}}=P^{\prime \prime} ; \frac{d V}{d \varphi}=V^{\prime} ; \frac{d^{2} V}{d \varphi^{2}}=V^{\prime \prime} .
$$

The first pressure derivative is:

$$
P^{\prime}=-n P \frac{V^{\prime}}{V}
$$

The second pressure derivative is:

$$
P^{\prime \prime}=-n P \frac{V^{\prime \prime}}{V}-(n+1) P^{\prime} \frac{V^{\prime}}{V} .
$$

In the fastest pressure growth point, the second pressure derivative is zero (appropriate angle (see fig. 3) $\varphi=\varphi_{m}$; let us identify also all values for $\varphi=$ $\varphi_{\mathrm{m}}$ by index $\langle\mathrm{m} »)$ :

$$
n P_{\mathrm{m}} V_{\mathrm{m}}^{\prime \prime}+(n+1) P_{\mathrm{m}}^{\prime} V_{\mathrm{m}}^{\prime}=0 \text {. }
$$

It means that in fastest pressure growth point the first pressure derivative by the $\mathrm{CA}$ is:

$$
P_{\mathrm{m}}^{\prime}=-P_{\mathrm{m}} \frac{n}{n+1} \frac{V_{\mathrm{m}}^{\prime \prime}}{V_{\mathrm{m}}^{\prime}} \text {. }
$$

From the other hand:

$$
P^{\prime}=-n P V^{\prime} / V \text {, }
$$

that means:

$$
-n P_{\mathrm{m}} \frac{V_{\mathrm{m}}^{\prime}}{V_{\mathrm{m}}}=-P_{\mathrm{m}} \frac{n}{n+1} \frac{V_{\mathrm{m}}^{\prime \prime}}{V_{\mathrm{m}}^{\prime}} .
$$

Finally we receive the following equation for fastest pressure growth point:

$$
V_{\mathrm{m}}=(n+1) \frac{\left(V_{\mathrm{m}}^{\prime}\right)^{2}}{V_{\mathrm{m}}^{\prime \prime}} \text {. }
$$

The second derivative of cylinder volume by the CA equals: 


$$
V_{\mathrm{m}}^{\prime \prime}=\left.\frac{d^{2} V}{d \varphi^{2}}\right|_{\varphi=\varphi_{\mathrm{m}}}=\frac{\pi D^{2} S}{8}\left(\begin{array}{l}
\cos \varphi_{\mathrm{m}}+ \\
+\frac{\sin ^{2} 2 \varphi_{\mathrm{m}}}{4 \Phi_{\mathrm{m}}^{1.5}}+ \\
+\frac{\cos 2 \varphi_{\mathrm{m}}}{\Phi_{\mathrm{m}}^{0.5}}
\end{array}\right)
$$

where $\Phi_{\mathrm{m}}=1 / \lambda^{2}-\sin ^{2} \varphi_{\mathrm{m}}$.

In the last formula $D$ - diameter of cylinder, $\mathrm{m}$; $S$ - piston stroke, $\mathrm{m} ; \lambda$ - ratio of connecting rod length $L$ to crank radius $R(\lambda=L / R=2 L / S$, $)$.

$V_{\mathrm{m}}$ and $V_{\mathrm{m}}^{\prime}$ are:

$$
\begin{aligned}
& V_{\mathrm{m}}=V_{c}+\frac{\pi D^{2} S}{8}\left(\begin{array}{l}
1+1 / \lambda-\cos \varphi_{\mathrm{m}}- \\
-\Phi_{\mathrm{m}}^{0.5}
\end{array}\right) \\
& V_{\mathrm{m}}^{\prime}=\left.\frac{d V}{d \varphi}\right|_{\varphi=\varphi_{\mathrm{m}}}=\frac{\pi D^{2} S}{8}\left(\begin{array}{l}
\sin \varphi_{\mathrm{m}}+ \\
+\frac{\sin 2 \varphi_{\mathrm{m}}}{2 \Phi_{\mathrm{m}}^{0.5}}
\end{array}\right)
\end{aligned}
$$

where $V_{c}$ - volume of compression chamber, $\mathrm{m}^{3}$.

It's clearly seen, that the expression (9) contains neither pressure nor pressure derivative. It means that for indicator diagram an angle, which corresponds to fastest pressure growth at the compression stroke, is invariant from pressure and depends only upon geometrical sizes of crank mechanism, compression ratio and polytropic coefficient.

By the meantime the manuals for modern diesel engines rather often do not contain the volume of combustion chamber $V_{c}$ (i.e. compression ratio $\varepsilon$ ). That's why in order to find angle $\varphi_{m}$ from (9), it must be supplemented by one more equation. In this case we obtain system of equations which helps us to find $V_{c}$ and $\varphi_{\mathrm{m}}$.

The most easy way is to use the formula:

$$
P_{\mathrm{m}}^{\prime}=-n P_{\mathrm{m}} V_{\mathrm{m}}^{\prime} / V_{\mathrm{m}} \text {. }
$$

In this case we receive the following system:

$$
\left\{\begin{array}{l}
V_{\mathrm{m}}=(n+1)\left(V_{\mathrm{m}}^{\prime}\right)^{2} / V_{\mathrm{m}}^{\prime \prime} \\
P_{\mathrm{m}}^{\prime}=-n P_{\mathrm{m}} V_{\mathrm{m}}^{\prime} / V_{\mathrm{m}}
\end{array}\right.
$$

It gives us:

$$
P_{\mathrm{m}}=-\frac{n+1}{n} P_{\mathrm{m}}^{\prime} \frac{V_{\mathrm{m}}^{\prime}}{V_{\mathrm{m}}^{\prime \prime}} .
$$

The angle $\varphi_{m}$ can be obtained from the last equation. The obtained value of $\varphi_{\mathrm{m}}$ then has to be implemented in the first equation in the system (14). It gives volume $V_{c}$.

The value of polytropic coefficient $n$ can be taken from data already known. For instance, we have $n \approx 1.37$ for quite wide range of medium- and slow-speed ship's diesels.

\section{APPLICATIONS}

\subsection{Calculation of angle $\varphi_{\mathrm{m}}$ and compression ratio}

Initial data for calculation $\varphi_{\mathrm{m}}$ and $\varepsilon$ are given in table 1 (see below): dimensions of crank mechanism $\left(D, S\right.$ and $\lambda$ ), values $P_{\mathrm{m}}$ and $P_{\mathrm{m}}^{\prime}$ (data for pressure were received from IMES $\mathrm{GmbH}$ ). Rotating speed of crankshaft while the engines' performances is also given there. The results of the calculations of $\varphi_{\mathrm{m}}$ and $\varepsilon$ are presented in table 2 . The polytropic coefficient was $n=1.37$. Figures which illustrate functional dependence of cylinder pressure and its derivative on CA for different engines are shown at the figures 4 and 5 .

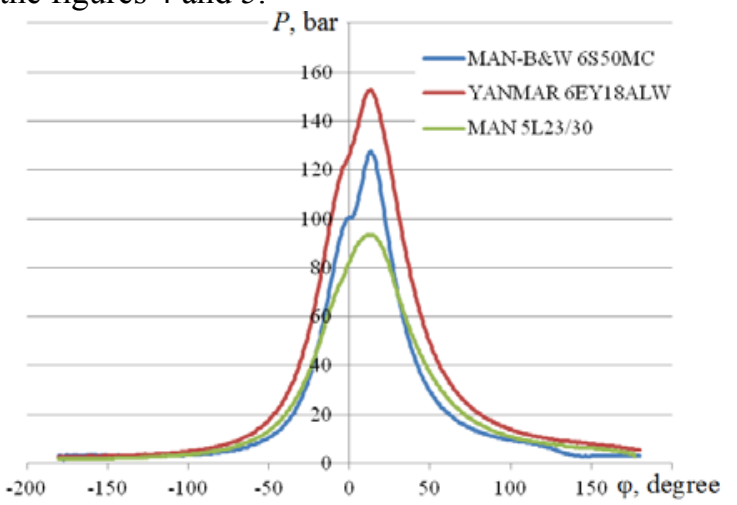

Fig. 4. Dependence of cylinder pressure upon the crank angle

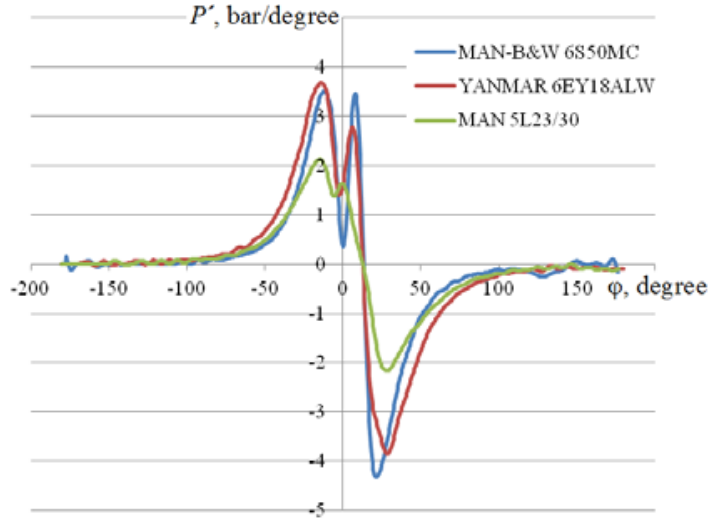

Fig. 5. Dependence of derivative of cylinder pressure on the crank angle

Comparison shows good coincidence of input and calculated values of angle $\varphi_{\mathrm{m}}$ and compression ratio $\varepsilon$.

\subsection{Change of angle $\varphi_{m}$ under different conditions}

Formula (9) gives us possibility to study the correlation between polytropic coefficient $n$ and angle $\varphi_{\mathrm{m}}$. This has been done for the engine YANMAR 6EY18ALW. Starting pressure of compression stroke was taken 2.92 bar for all cases. Results of modelling are shown at fig. 6 and table 3 .

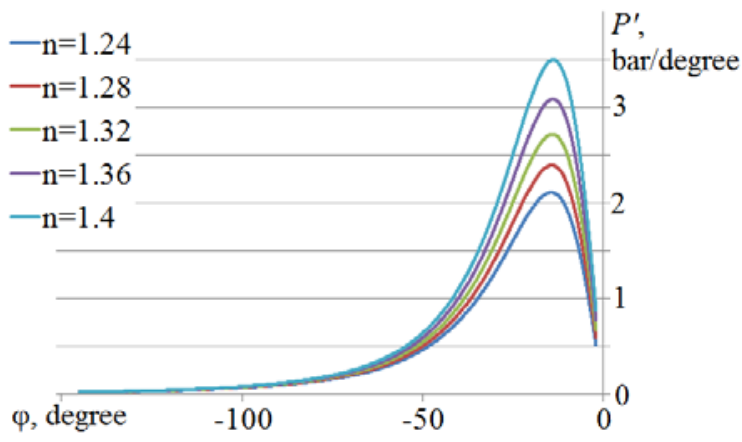

Fig. 6. Cylinder pressure derivative dependence upon the crank angle during compression stroke under variable polytropic coefficient 
Yeryganov O, Varbanets R.: Features of the fastest pressure growth point during compression stroke

Table 1. Initial data for calculating angle $\varphi_{\mathrm{m}}$ and compression ratio $\varepsilon$

\begin{tabular}{|c|c|c|c|c|c|c|c|}
\hline \multirow{2}{*}{$\begin{array}{l}\text { Name of } \\
\text { engine }\end{array}$} & \multicolumn{5}{|c|}{ Engine's data } & \multirow{2}{*}{$\begin{array}{c}\text { Pressure } \\
P_{\mathrm{m}} \text {, bar }\end{array}$} & \multirow{2}{*}{$\begin{array}{c}\text { Angle derivative of } \\
\text { pressure } P_{\mathrm{m}}^{\prime}, \\
\text { bar/degree }\end{array}$} \\
\hline & Stroke & RPM & $D, \mathrm{~m}$ & $S, \mathrm{~m}$ & $\lambda$ & & \\
\hline $\begin{array}{c}\text { MAN-B\&W } \\
\text { 6S50MC }\end{array}$ & 2 & 100 & 0.5 & 2.0 & 0.4878 & 72.18 & 3.501 \\
\hline $\begin{array}{l}\text { YANMAR } \\
\text { 6EY18ALW }\end{array}$ & 4 & 900 & 0.18 & 0.28 & 0.25926 & 90.49 & 3.678 \\
\hline $\begin{array}{c}\text { MAN } \\
\text { 5L23/30 }\end{array}$ & 4 & 720 & 0.225 & 0.3 & 0.25 & 56.86 & 2.127 \\
\hline
\end{tabular}

Table 2. Comparison of calculated and input data for angle $\varphi_{\mathrm{m}}$ and compression ratio $\varepsilon$

\begin{tabular}{|c|c|c|c|c|}
\hline \multirow{2}{*}{$\begin{array}{c}\text { Name of } \\
\text { engine }\end{array}$} & \multicolumn{2}{|c|}{ Compression ratio $\varepsilon$} & \multicolumn{2}{c|}{ Angle $\varphi_{\mathrm{m}}$ (before TDC), degree } \\
\cline { 2 - 5 } & calculation & design & calculation & experiment \\
\hline MAN-B\&W 6S50MC & 18.1 & 18.1 & -11.6 & -11.8 \\
\hline YANMAR 6EY18ALW & 15.4 & 15 & -13.8 & -14 \\
\hline MAN 5L23/30 & 13.3 & 13 & -14.9 & -15 \\
\hline
\end{tabular}

Table 3. Caclulated angle $\varphi_{\mathrm{m}}$ with variable polytropic coefficient

\begin{tabular}{|c|c|}
\hline Polytropic coefficient $\mathrm{n}$ & Angle $\varphi_{\mathrm{m}}$, degree \\
\hline 1.24 & -14.5 \\
\hline 1.28 & -14.3 \\
\hline 1.32 & -14.1 \\
\hline 1.36 & -14.0 \\
\hline 1.4 & -13.8 \\
\hline
\end{tabular}

It can be seen that in the working range $(n=$ $1.3 \ldots 1.4)$ the angle $\varphi_{m}$ varies within the limits $14.1^{\circ} \ldots-13.8^{\circ}$.

We also checked numerically for the same engine, whether the angle $\varphi_{\mathrm{m}}$ depends on the pressure (see formula (9)). For this purpose compression stroke was calculated for different starting pressures $P_{0}$. In these cases polytropic coefficient $n=1.37$. Results of the modelling are given at fig. 7 and in table 4 .

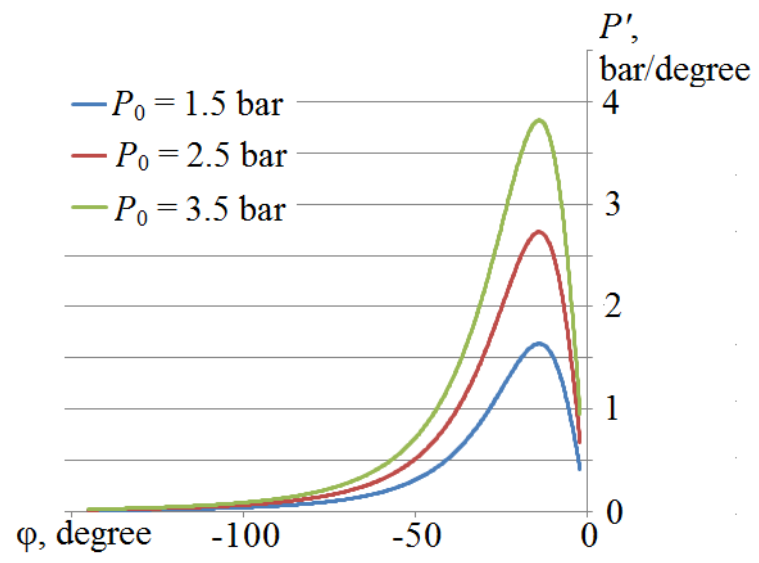

Fig. 7. Dependence of the cylinder pressure derivative upon the crank angle during compression stroke under variable starting pressure
Table 4. Calculated angle $\varphi_{\mathrm{m}}$ with variable starting

\begin{tabular}{|c|c|}
\hline \multicolumn{2}{|c}{ pressure } \\
\hline Starting pressure, bar & Angle $\varphi_{\mathrm{m}}$, degree \\
\hline 1.5 & -13.9 \\
\hline 2.5 & -13.9 \\
\hline 3.5 & -13.9 \\
\hline
\end{tabular}

In order to show correlation between the sizes of crank mechanism and angle $\varphi_{\mathrm{m}}$, compression stroke was modeled for different coefficient $\lambda$ (i.e. for different length $L$ of connecting rod). For this case starting pressure $P_{0}=2.92$ bar, polytropic coefficient $n=1.37$. The rest data $(D, S, \varepsilon)$ was taken for engine YANMAR as per tables 1 and 2 . Results of the modelling are shown at fig. 8 and in table 5.

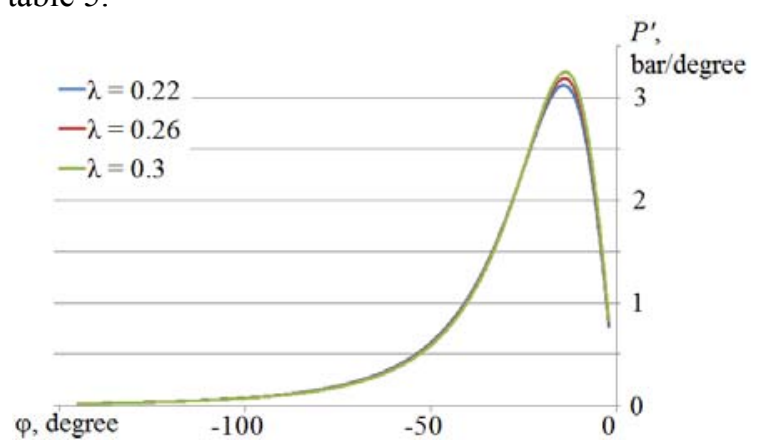

Fig. 8. Dependence of the cylinder pressure derivative upon the crank angle during compression stroke with variable coefficient $\lambda$

Table 5. Calculated angle $\varphi_{\mathrm{m}}$ with variable coefficient $\lambda$

\begin{tabular}{|c|c|}
\hline Coefficient $\lambda$ & Angle $\varphi_{\mathrm{m}}$, degree \\
\hline 0.22 & -14.1 \\
\hline 0.26 & -13.9 \\
\hline 0.3 & -13.7 \\
\hline
\end{tabular}

To determine if angle $\varphi_{\mathrm{m}}$ depends on actual compression ratio, it's necessary to search curve $P^{\prime}$ for different angle of compression stroke beginning (while volume of compression chamber $V_{c}$ remains 
the same). The modeling was conducted for engine YANMAR (see tables 1 and 2). Polytropic coefficient $n=1.37$. In all cases pressure in the beginning of compression was equal 2.92 bar. Results of modeling are represented at fig. 9 and table 6 .

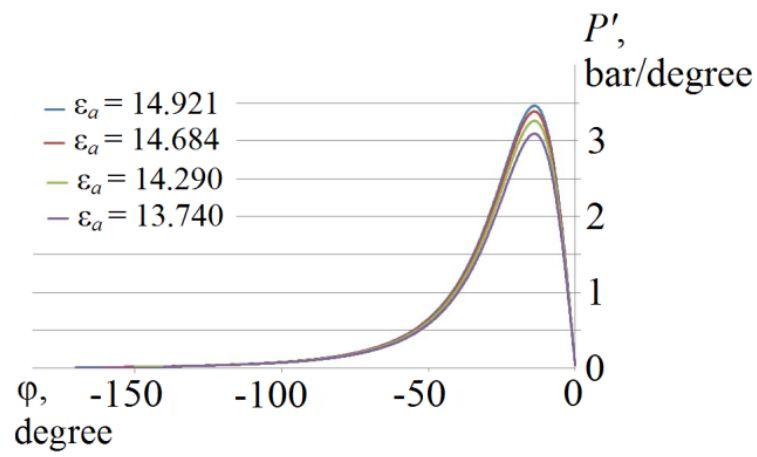

Fig. 9. Dependence of the cylinder pressure derivative upon the crank angle during compression stroke under variable actual compression ratio

Table 6. Calculated angle $\varphi_{\mathrm{m}}$ with variable actual compression ratio

\begin{tabular}{|c|c|c|}
\hline $\begin{array}{c}\text { Beginning of } \\
\text { compression, } \\
\text { degree before TDC }\end{array}$ & $\begin{array}{c}\text { Actual } \\
\text { compression } \\
\text { ratio } \varepsilon_{a}\end{array}$ & $\begin{array}{c}\text { Angle } \varphi_{\mathrm{m}}, \\
\text { degree }\end{array}$ \\
\hline-170 & 14.921 & -13.9 \\
\hline-160 & 14.684 & -13.9 \\
\hline-150 & 14.290 & -13.9 \\
\hline-140 & 13.740 & -13.9 \\
\hline
\end{tabular}

Results of the modelling show that the position of fastest pressure growth point at the indicator diagram is almost steady, and under usual conditions varies within the narrow range (around $\left.0.2^{\circ}\right)$.

\subsection{TDC determination by solution of equation} $\boldsymbol{P}^{\prime}=\mathbf{0}$

It was mentioned already in chapter 2.1 , that in case of absence of combustion and zero thermodynamic displacement $\left(\delta \varphi_{T}=0\right)$, the first pressure derivative of the $\mathrm{CA}$ in TDC equals zero $[4-6,8,14]$ :

$$
P^{\prime}\left(\varphi-\delta \varphi_{T}\right)=P^{\prime}(\theta)=0 .
$$

The value of $\delta \varphi_{T}$ depends on type of diesel and its cooling.

Using results which were received in chapter 2.3 , it's possible to find the angle $\varphi_{\mathrm{m}}$ between TDC and maximum of first pressure derivative. The value $\varphi_{\mathrm{m}}$ found in such a way can be considered as a first approximation for solution of equation $P^{\prime}(\theta)$ $=0$, which results acceptable accuracy for TDC determination. The first approximation is necessary in order to speed up solution of equation $P^{\prime}(\theta)=0$ and synchronize the data (i.e. transfer pressure them from the functions of time into the CA function). This transfer is important as further calculations of parameters are conducted relatively CA. As engine's settings are given also in CA, the synchronization is the first step on which depends the accuracy of diagnostics.

The speed of synchronization is extremely important for real-/pseudo-real-time calculations of indicator diagram. The values of mean indicating pressure and mean indicating power can be considered as final result of such calculations [14].

The expression for rate of pressure change at compression stroke is written based on assumption of polytropic compression (see chapter 2.3).

$$
\frac{d P}{d \theta}=-n P_{a} \frac{V_{a}}{(V(\theta))^{n+1}} \cdot \frac{d V(\theta)}{d \theta},
$$

where $P_{a}, V_{a}$ - pressure and cylinder volume at the beginning of compression stroke;

$V(\theta)=V\left(\varphi-\delta \varphi_{T}\right)-$ current volume of cylinder.

The solution of equations (16) and (17) gives the improved value of TDC position. The thermodynamic displacement $\delta \varphi_{\mathrm{T}}$ can be found using Woschni (or similar) formula [14].

\section{CONCLUSION}

Results of calculations show that the described method can be used for preliminary calculating of TDC location in indicator diagram and compression ratio $\varepsilon$. It has been numerically proved that position of fastest pressure growth point at compression stroke is invariant upon the pressure and its derivative. Actual presence of this point in the indicator diagram is the main advantage of the suggested method of TDC calculation, as in this case it is not necessary to find additional conditions and restrictions.

Generally speaking, the using of algorithmic TDC determination methods makes the diagnostics faster and available for any diesel engine under the load. Besides that, it gives the possibility to take automatically into account errors caused by: 1) the torsional vibrations or crank mechanism backlashes; 2) the twisting of the crankshaft (which is very important for cylinders far from flywheel); 3 ) the velocity of pressure wave in the indicator cock channel (displacement caused by this can achieve few degrees for medium- and high-speed diesels [14]).

\section{REFERENCES}

1. Brown WL. Methods for evaluating requirements and errors in cylinder pressure measurement. SAE Paper $\mathrm{N}^{\circ} 670008$. https://doi.org/10.4271/670008

2. Brunt M., Emtage A. Evaluation of IMEP Routines and Analysis Errors SAE Paper $\mathrm{N}^{\circ} 960609$. https://doi.org/10.4271/960609

3. Cylinder pressure sensors: http://imes.de/htt-04.html

4. Ding Y, Stapersma D, Knoll H, Grimmelius HT. Characterising heat release in a diesel engine: $A$ comparison between Seiliger process and Vibe model. In Proceedings of the 28th CIMAC World Congress, Bergen, Norway, 2011. 
5. Ding Y, Stapersma D, Grimmelius HT. Cylinder process simulation with heat release analysis in diesel engine. Conference: Power and Energy Engineering Conference, 2009. APPEEC 2009. Asia-Pacific. https://doi.org/10.1109/APPEEC.2009.4918248

6. Ding Y, Stapersma D, Grimmelius HT. Using parametrized finite combustion stage models to characterize combustion in diesel engines. Energy \& Fuels, 2013: 7099-7106.

7. Hribernik A. Statistical determination of correlation between pressure and crankshaft angle during indication of combustion engines. SAE Paper $\mathrm{N}^{\circ}$ 982541. https://doi.org/10.4271/982541

8. Lapuerta M, Armas O, Bermúdez V. Sensitivity of diesel engine thermodynamic cycle calculation to measurement errors and estimated parameters. Applied Thermal Engineering, 2000; 20(9): 843-861. https://doi.org/10.1016/S1359-4311(99)00069-1

9. Motohiro T, Shigeji T. Performance and combustion analysis of high-speed diesel engine in fast ferry under normal service condition. CIMAC Congress Kyoto. 2004; 43: 1-8.

10. Otnes RK, Enochson L. Applied time series analysis. New York: Wiley, 1978: 449

11. Pinchon P. Thermodynamic calibration of top dead center in piston engines. Oil \& Gas Science and Technology - Rev. IFP, 2006; 39(1): 93-111. https://doi.org/10.2516/ogst:1984007

12. Smith SW. The scientist and engineer's guide to digital signal processing. - Second Edition. - SanDiego: California Technical Publishing, 1999.

13. Staś M. An universally applicable thermodynamic method for T.D.C. determination. SAE Technical Paper 2000-01-0561, 2000. https://doi.org/10.4271/2000-01-0561

14. Stapersma D. Diesel engines, Volume 1: Performance analysis. Royal Netherlands Naval College, 2002.

15. Tazerout M, Le Corre O, Rousseau S. TDC determination in IC engines based on the thermodynamic analysis of the temperature-entropy diagram. SAE Technical Paper 1999-01-1489, 1999. https://doi.org/10.4271/1999-01-1489

16. Varbanets R, Karianskiy A. Analyse of marine diesel engine performance. Journal of Polish CIMAC. Energetic Aspects. Gdansk: Faculty of Ocean Engineering and Ship Technology Gdansk University of Technology. 2012; 7(1): 269-275.

Received 2018-01-16

Accepted 2018-04-04

Available online 2018-04-09

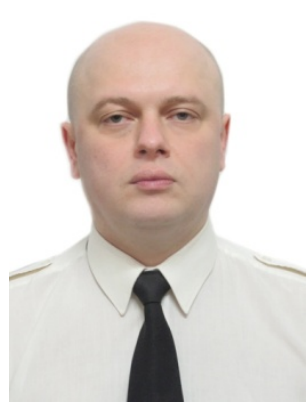

Oleksiy YERYGANOV

has received his $\mathrm{PhD}$ in

Technical Sciences in 2010.

Currently he is assistant professor of "The Marine Power Plants and Technical Operation" Department in Odessa National Maritime University. He holds also Chief Engineer license for ships powered by internal combustion engines.

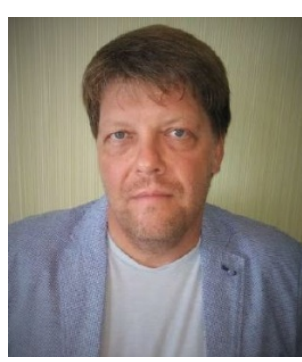

Roman VARBANETS

defended his doctoral dissertation in Technical Sciences in 2010. Currently he is professor Head of "The Marine Power Plants and Technical Operation" department in Odessa National Maritime University. IMES $\mathrm{GmbH}$ mathematics consultant. 